Session VI

ORIGIN AND EVOLUTION 


\title{
OBSERVATIONAL ASPECTS OF THE EVOLUTION \\ OF PLANETARY NEBULAE AND THEIR CENTRAL STARS
}

\author{
C. R. O'DELL \\ (The Yerkes Observatory, Williams Bay, Wisc., U.S.A.)
}

\begin{abstract}
The greatest activity in the area of evolution of the central stars of the planetary nebulae has occurred in the last two decades, although there have been significant contributions by many workers of considerable insight ever since the early studies at the Lick Observatory demonstrated the physical nature of this class of nebula. We shall try to draw together here the many facets of the present picture of this rapidly developing and highly significant phase of stellar evolution. Unfortunately, the picture is not at all as complete as one would like, and there remain several points of scientific contention; however, we should like to present the results of several investigators.
\end{abstract} A certain amount of redundancy with previous papers given prior will be necessary, but we shall endeavor to treat these topics briefly and in the context of the evolution of the nebulae and their stars.

Although this is a very rapid state of stellar evolution, the study of the changes in the nebulae and their stars is singularly favored. This is true for simple discovery because of their emission-line nature, which yields to surveys at relatively high spectral purity by filters and objective-prism spectroscopy techniques, that has resulted in the discovery and identification of a relatively complete sample in the solar neighborhood and a significant fraction of those objects in as far as the Galactic centre. The astrophysical process of changing the abundant high-energy ultraviolet central star photons into optical emission lines through photo-ionization followed by recombination and collisional excitation of metastable levels means that we have tools for the determination of the stellar and nebular temperatures, masses, luminosities and distances. This feature of conversion of photons from the spectral region of highest luminosity into readily observable photons is the greatest advantage of these systems and has made it possible to say more about the planetary nebula systems than any other advanced evolutionary system, except perhaps for the white dwarfs.

The discussion of the evolution of these systems depends primarily upon two features: (1) the determination of central star temperatures (since temperatures and the bolometric corrections are large), and (2) the determination of the distances to the nebulae (in order to find the apparent luminosities). We would like to briefly discuss these two areas and how they affect the present evolutionary picture. 


\section{Stellar Temperatures}

Stellar temperatures may be inferred from spectroscopic characteristics and from the astrophysical Zanstra (1926) method. We shall first consider the former.

With a very few exceptions the assigned spectral types are quite early, falling into the $\mathrm{O}$ classification. There is a broad range of subdivisions, however; these are listed in Table 1 .

\section{Table 1}

Stellar Types

Wolf-Rayet

O types

Of types

Continuum
Spectral Characteristics

Broad emission lines Of, $\mathrm{He}$ II, CIII, etc.

Hydrogen, $\mathrm{He} \mathrm{I}$ and $\mathrm{He} I \mathrm{I}$ in absorption

Narrow emission of CiII, N III, HeII, etc.

No emission or absorption features seen at the low dispersions employed

The division into types is by no means as clear cut and simple as this table would indicate. The absorption lines in the $O$ types range from strong, deep, and narrow lines to those verging upon the continuum classification because of the low central depth and great width of their lines. The selectively brighter (apparently and intrinsically) Wolf-Rayet, strong line O's and Of types were studied early, and it was noted by Aller (1956) and others that a large range of line widths exists both in emission and absorption. Greenstein and Minkowski (1964) published the results of a study selectively oriented to the central stars associated with the lower surface brightness nebulae. In that investigation it was seen that generally the hydrogen lines were broad and weak and that the degree of excitation was quite high, even finding strong $\mathrm{O} v$ in absorption and $\mathrm{OVI}$ in emission in NGC 246. The crude selection effects of first studying the high surface brightness, classical nebulae and then those of lower surface brightness give some indication of different spectral characteristics associated with various nebulae. This tendency is much more clearly delineated when one considers the final absolute luminosity-temperature tracks that are derived. However, there is no strong evidence that there exists a single valued, monotonic sequence of spectral types, although there does exist the general feature of the most luminous central stars usually having stronger and deeper absorption lines - often associated with broad emission lines similar to the Of and Wolf-Rayet stars. The intrinsically less luminous stars have selectively broader and shallower absorption lines (or even none at all) than do the earlier spectral types and show emission and absorption features of high states of ionization. The observational difficulties in obtaining adequate spectra of these stars are considerable, arising from the superimposed strong nebular emission and from the faintness of the stars themselves. There remains much to be done in this area for observers possessing adequate equipment.

More quantitative measures of the stellar temperatures can be derived from the original method developed by Zanstra (1926) and its variations. In its simplest form, 
this theory derives from the supposition that all stellar photons capable of the ionization of a hydrogen atom will do so and that upon recombination and cascade of the ionized and excited hydrogen atoms, a sufficient optical depth in Lyman-line radiation prevails so that each original photo-ionization is ultimately followed by the emission of a Balmer line or continuum photon. This condition is stated in the first equation.

$N($ Stellar Lyman Continuum Photons $) \geq N($ Nebular Balmer Photons),

where the inequality arises if the nebula is not optically thick to the Lyman continuum. Under the assumption of knowledge of the continuum flux distribution of the central star, one can then derive the functional relation between the stellar and nebular brightness

$$
m_{\mathrm{star}}+2 \cdot 5 \log F(\mathrm{H} \beta) \leqslant 2 \cdot 5 \log F\left(T_{\mathrm{s}}\right)+\text { Constant } .
$$

This method is in essence a very broad base-line color index, where the emissionline flux in $\mathrm{H} \beta$ is used as a quantitative measure of the amount of ultraviolet stellar radiation. From the resultant temperatures and continuing the assumption that the flux distribution is known (usually a blackbody), it is a simple matter to calculate bolometric corrections (O'Dell, 1963a).

This same basic method can also be extended to other abundant elements, such as HeI and HeII, as was originally done by Würm and Singer (1952) and more recently by Harman and Seaton (1966). The primary advantage lies with HeII since a nebula becomes optically thin to it at a much later stage in its expansion than does hydrogen. This means that He II Zanstra temperatures can be used rigorously after the hydrogen temperatures have become lower limits. The question of the validity of the usual assumption of a stellar atmosphere emitting as a blackbody must, of course, arise; since it is well known that in the population-I early-type stars very severe depressions of the flux at the Lyman discontinuity can occur. This can be easily tested by reference to the detailed model atmospheres calculated by Gebbie and Seaton (1963) and by Böhm and Deinzer (1965). The latter models, which were calculated for temperatures and surface gravities relevant to the early evolutionary picture indicate that the deviations from the black-body assumption in derived temperatures and absolute luminosities are not extremely large compared to the other uncertainties involved. The necessary corrections are in the sense that the effective temperatures should be larger than derived and will be most important for the coolest central stars.

\section{Distances to the Nebulae}

The distance to the planetary nebulae has been the subject of considerable discussion during the last several years (Shklovsky, 1956; O'Dell, 1962; Seaton, 1966; Abell, 1966) owing to an increased appreciation of the importance of this parameter and the existence of the accurate emission-line brightnesses coming from the work of Liller (1955) 
and of Osterbrock and his students (Capriotti and Daub, 1960; Collins et al., 1961; O'Dell, 1962, 1963b). Space does not permit a discussion of the difficulties of the calibration of the distance scales, but we shall briefly review the basic considerations of this problem. It is now widely recognized that planetary nebulae exist in both the completely ionized and partially ionized states, depending upon their ultraviolet luminosity and nebular size and density. The method of distance determination to be applied depends upon the condition of ionization. The basic equations describing the observable characteristics of a planetary nebula are given below:

$$
\begin{gathered}
\mathscr{F}(\mathrm{H} \beta)=1 \cdot 33 \pi S^{3} \varepsilon N_{\mathrm{c}}^{2} \alpha E(\mathrm{H} \beta) \\
\Phi=S / D \\
F(\mathrm{H} \beta)=F(\mathrm{H} \beta) / 4 \pi D^{2},
\end{gathered}
$$

where $\mathscr{F}$ is the total $\mathrm{H} \beta$ luminosity of a nebula of linear radius $S$, density $N_{\mathrm{e}}$, and fraction of volume filled by material $\varepsilon . N_{\mathrm{e}}^{2} \alpha E(\mathrm{H} \beta)$ is the volume emissivity in $\mathrm{H} \beta$. The observed angular radius $(\Phi)$ and observed flux $F(\mathrm{H} \beta)$ are, of course, related to the distance $D$, so that one can write an expression for the linear radius

$$
S=3 F(\mathrm{H} \beta) / \Phi^{2} \varepsilon \alpha E(\mathrm{H} \beta) N_{\mathrm{e}}^{2} .
$$

To apply this method one needs to know the flux in $\mathrm{H} \beta$, the angular size, the approximate electron temperature, the filling factor (estimated from photographs) and the density (usually found from forbidden-line data). This simple expression is rigorous and universally applicable, and has most widely been used by Seaton (1966) and his collaborators. There are no assumptions made of the Lyman-continuum optical depth, this being a great advantage of the method. However, this method has in practice been applied only to the highest surface brightness nebulae because of the difficulty of obtaining for the lower surface brightness nebulae the emission-line ratios necessary for the determination of the electron densities. The basic limitation of this method is the strong effects of filamentary structure on the resultant nebular distance and hence stellar luminosity. The filling factor used by Seaton (1966) was based upon examination of direct photographs and primarily considered the large-scale geometric distribution. This filling factor may be a considerable overestimate, however, because of the possible presence of unresolved filamentary structure, something often seen in very good photographs and indicated from analysis of forbidden-line ratios (Seaton and Osterbrock, 1957). The derived luminosity by this method is also very sensitive to each observable parameter:

$$
L_{\mathrm{star}}=\left[36 \pi / \alpha^{2} E^{2}(\mathrm{H} \beta)^{2}\right]\left[l_{\mathrm{star}} F(\mathrm{H} \beta)^{2} / \phi^{6} \varepsilon^{2} N_{\mathrm{e}}^{4}\right]
$$

For conditions of density not so large that collisional de-excitation is important, the density derived trom forbidden-line ratios (usually the [OIII] $\lambda 3727$ doublet) are weighted towards being representative of the denser filaments since the emissivity 
increases as the square of the density. This would mean that errors would arise in the use of the luminosity relation above since a large part of the nebular emission might come from the low-density regions. The magnitude of such effects can be seen from considering a two-state nebula where $\left(N_{\text {high }} / N_{\text {low }}\right)^{2} \gg 1$ and $\varepsilon_{\text {low }} / \varepsilon_{\text {high }}>1$, a model suggested by Seaton and Osterbrock's measurements of NGC 7027. In this case the correction factor in the luminosity expression becomes $\left(\varepsilon_{\text {low }} / \varepsilon_{\text {high }}\right)^{2}$, e.g., in a nebula where three times as much volume is filled by the low-density material as for the high density, the true luminosity will be nine times larger than that derived from the elementary application of this method. The postulation of such filaments as general features of the nebulae is somewhat $a d$ hoc in nature and may not generally be applicable; however, the luminosities derived by this method should probably be considered as lower limits.

It is possible (under much more restrictive conditions) to determine the distances of planetary nebulae for which the densities are not known. If the nebulae are completely ionized, the ionized (visible) radius will equal that of the gas itself $(S=R)$ and the mass of the nebula is given by

$$
M=1 \cdot 33 \pi R^{3} \varepsilon N_{\mathrm{e}} m_{\mathrm{H}} .
$$

Combining this expression with those given before and eliminating the electron density one can derive the generalized expression for optically thin nebulae:

$$
R=\left[\frac{3 \alpha E(\mathrm{H} \beta)}{16 \pi^{2} m_{\mathrm{H}}^{2}} \cdot \frac{M}{\varepsilon} \cdot \frac{\phi^{2}}{F(\mathrm{H} \beta)}\right]^{1 / 5},
$$

which was first used extensively in only slightly different form by Šklovsky (1956). This method requires a determination of the factor $\left(M^{2} / \varepsilon\right)^{1 / 5}$. The usual assumption has been that the variations in this quantity are sufficiently small that an average value can be determined from calibration procedures on a few objects, and this value can then be applied to all other optically thin nebulae. O'Dell (1962) has tested this assumption of constancy by examination of the density-surface brightness relation expected, while Seaton (1966) has noted a leveling-off of a mass function as one passes from optically thick to optically thin nebulae. Seaton has employed this apparent upper limit for the mass derived from a few optically thin planetaries of known electron densities for calibration of his distance scale, while O'Dell has relied upon the average calibration found from the expansion of NGC 6720, the binary nature of the central star of NGC 246 and statistical parallaxes. Seaton's published distances are $45 \%$ larger than O'Dell's for optically thin nebulae. If one tries to apply the optically thin method to an optically thick nebula, an overestimate of the distance will result a fact applied by Minkowski by using both methods on a single object to discriminate thick and thin nebulae. The most useful criterion for discrimination between types is the appearance of neutral oxygen forbidden lines that arise in the interface of ionization while relative strengths of HeIII, HeII and $\mathrm{H}$ II lines can be helpful. Although by 
no means precise, it is possible to obtain distances good to a factor of 2 for most individual planetary nebulae at this time.

\section{Resultant Picture of the Evolution}

The evolution is seen in the regions that are populated in a logarithmic plot of stellar temperature and luminosity. Actual evolution through this populated region is indicated by the fact that the mean size of the nebular shell changes from sub-region to sub-region. Since it is reasonable to assume that the nebulae are all rather similar, and it is known that the material is in expansion, one can trace the sense of the evolution and determine its time-scale. In order to tie the entire picture together it is necessary to use the results of several approaches, depending upon the conditions of optical depth; we shall, therefore, discuss three sub-regions of stellar luminosity separately.

A. The earliest stages are characterized by a relatively high luminosity, temperatures not as high as found later and very dense surrounding nebulae. The latter characteristics dominate to make this a region where the nebulae are selectively optically thick to ionizing radiation, a feature brought out in Galactic planetaries by the presence of the [OI] lines. There are two main sources of material for this region - the distances of Seaton (1966) on the optically thick scale plus hydrogen Zanstra temperatures for Galactic objects, and the study in the Magellanic Clouds by Miss Webster (1967). Unfortunately, there are relatively few optically thick Galactic planetaries with low central star temperatures so that it is possible to easily misinterpret the form of the early evolution. The data by Miss Webster in the Magellanic Clouds is quite homogeneous due to the constant and known distance to the nebulae and she has derived Zanstra temperatures in the usual manner. In this case the cool end of the sequence is relatively well populated and it is possible to obtain a clearer picture. A final object falling into this region is the planetary nebula $\mathrm{K} 648$ in the globular cluster M 15 (O'Dell et al., 1964) whose distance is accurately known (for these purposes).

There seems to be little question about a general rise in stellar luminosity with increasing temperature in this phase, even after allowance for the many sources of uncertainty. The only remaining controversy is the degree to which the planetaries hook upwards. Seaton has argued that his two points at $\log L$ about 2 indicates a very strong hook while the Magellanic Cloud data of Miss Webster and K 648 would indicate that the rise occurs, but that there is essentially no other evidence for its occurring before a logarithmic luminosity of $+3 \cdot 5$. A very strong hook might be more compatible with the idealized models of Vila (1966), Shaviv (1967), L'Ecuyer (1966), Rose (1966) and Bautz (1968), although models such as some of those of Deinzer (1967) are flatter in this region. Although there has not been a thorough, homogeneous statistical approach to the spectral study of these central stars, there is obviously a very large, if not nearly complete, fraction that display Wolf-Rayet features. 
The existence of $\mathrm{W}-\mathrm{R}$ features in the spectra of stars has often been associated with mass loss, and it is relevant to ask if there is evidence of continued mass loss from the central stars to the nebulae during this period. This question is very hard to settle since one can determine a mass only for the ionized region, which is continuously changing as the mean density decreases. The disappearance of the $\mathrm{W}-\mathrm{R}$ features at hotter, later stages argues that mass loss cannot continue much beyond the time when a typical object is identified as a planetary nebula system. The radius range in this earliest stage is about $0 \cdot 04-0 \cdot 12$ parsecs.

B. What we shall designate here as the intermediate stages those nebulae characterized by a rather well-defined low optical depth in the hydrogen Lyman continuum. The neutral oxygen lines have disappeared and the constant mass method can be employed to find the distances. The absorption lines in the stars have become weaker and broader and the broad emission features are usually absent. The very fact that allows us to obtain the relatively good distances means that the hydrogen Zanstra temperatures will only be lower limits. Seaton (1966) has avoided this difficulty by adopting criteria based upon relative line strengths to pick out those objects that remain optically thick to $\mathrm{He}$, so that the helium Zanstra temperature can provide a rigorous value. We show in the illustration the results of inclusion of these stars, where one can see that the range of temperatures is not extremely large. Across this region there appears to be a general decrease in luminosity as the nebular radius increases from 0.12 to 0.25 parsecs. There are many more nebulae that have already been identified and probably fall into this class, but they do not appear here because the test for the validity of the helium Zanstra temperature requires emission-line ratios, which are not known for many of these objects, since this is a region where the surface brightness of the nebula is decreasing as the fifth power of the nebular radius. In actuality this region is probably populated as heavily as the earliest stage.

C. In the late stages the radii of the gas shells are over 0.25 parsecs due to continued expansion of the material. Since the stars seem to have dropped significantly in luminosity, but have not changed greatly in temperature, the effective stellar radii are significantly smaller than in the prior two stages; thus, the radiation dilution has increased. At the same time the mean nebular gas density is continuously decreasing, which would increase the volume ionized; therefore, we have competing mechanisms for determining the optical depth in the Lyman continuum, a fact pointed out by Seaton (1966). The total number of ionizing photons emitted by the star per unit time can easily be related to the ionization radius, as can the luminosity of the star and its temperature and radius. Since there exists an average relation between the nebular density and the total gas radius, we can derive a general expression for the ratio of the ionization radius and the gas radius,

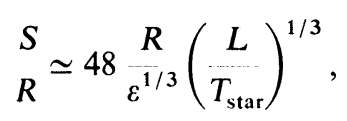


which will be a function of the shell size, and the stellar temperature and luminosity. This means that the transition between complete and partial ionization will be described by a straight line of small slope in our logarithmic temperature-luminosity plot, with the ordinate shifted according to the total gas radius. That is, at a given point on the diagram all nebulae smaller than the transition size will be optically thick. By inserting numerical values for the derived sizes one can see that a few of the very low surface brightness nebulae are probably optically thick and many more fall into a marginal zone.

We plot in Figure 1 the hydrogen Zanstra temperatures and the luminosities based on the optically thin distance scale - which involves a logical inconsistency. However, the temperatures are probably rather close while the luminosities are not critically dependent upon the exact ionized mass due to the low power of the mass dependence. Actually, there is a very interesting problem all along the optically thin sequence. This is the fact that the hydrogen Zanstra temperatures never become greatly different from the true values while one would naively theoretically expect that the lower limit would become much lower than the true value as the transition to being completely ionized occurs. Capriotti has derived quantitative values for the optical depth in the Lyman continuum by reference to the He I Zanstra temperatures and has found values of the optical depth of less than one in many cases although an abrupt drop of several orders of magnitude as might be expected is not present.

The effect of underestimating the Zanstra temperatures in this region would move the stellar points up and to the left while the application of the wrong method of distance determination would lower the points in the ordinate. Definitely, there are uncertainties in the positions of the individual points in this region, but these are probably small compared with the intrinsic range of positions that are observed. The occupied region is reasonably well defined in our luminosity-temperature plot. The points shown on the figure correspond only to those known systems that, from the sizes of their nebular envelopes, fall close to regaining the condition of significant hydrogen Lyman continuum optical depth and hence populates the lower region to the exclusion of the upper - within the classification of the late stage of development.

It is very important to note that there seems to be a rather continuous sequence of conditions present here, carrying from the high-luminosity stars down through those close to the solar luminosity. The lowest-luminosity stars are comparable in luminosity, temperature, and spectrum to some of the hottest white dwarfs that have been studied. It is altogether reasonable to think that a progression of nebular sizes represents a progression in age since the appearance and nature of the objects are very similar. This enables one to derive an approximate time-scale for passing through the various stages discussed here. Taking as a typical maximum size radius the value 0.7 parsecs and an average expansion velocity of $20 \mathrm{~km} / \mathrm{sec}$, we find a time-scale of about 35000 years. This low value, indicating very rapid evolution of the illuminating stars is 


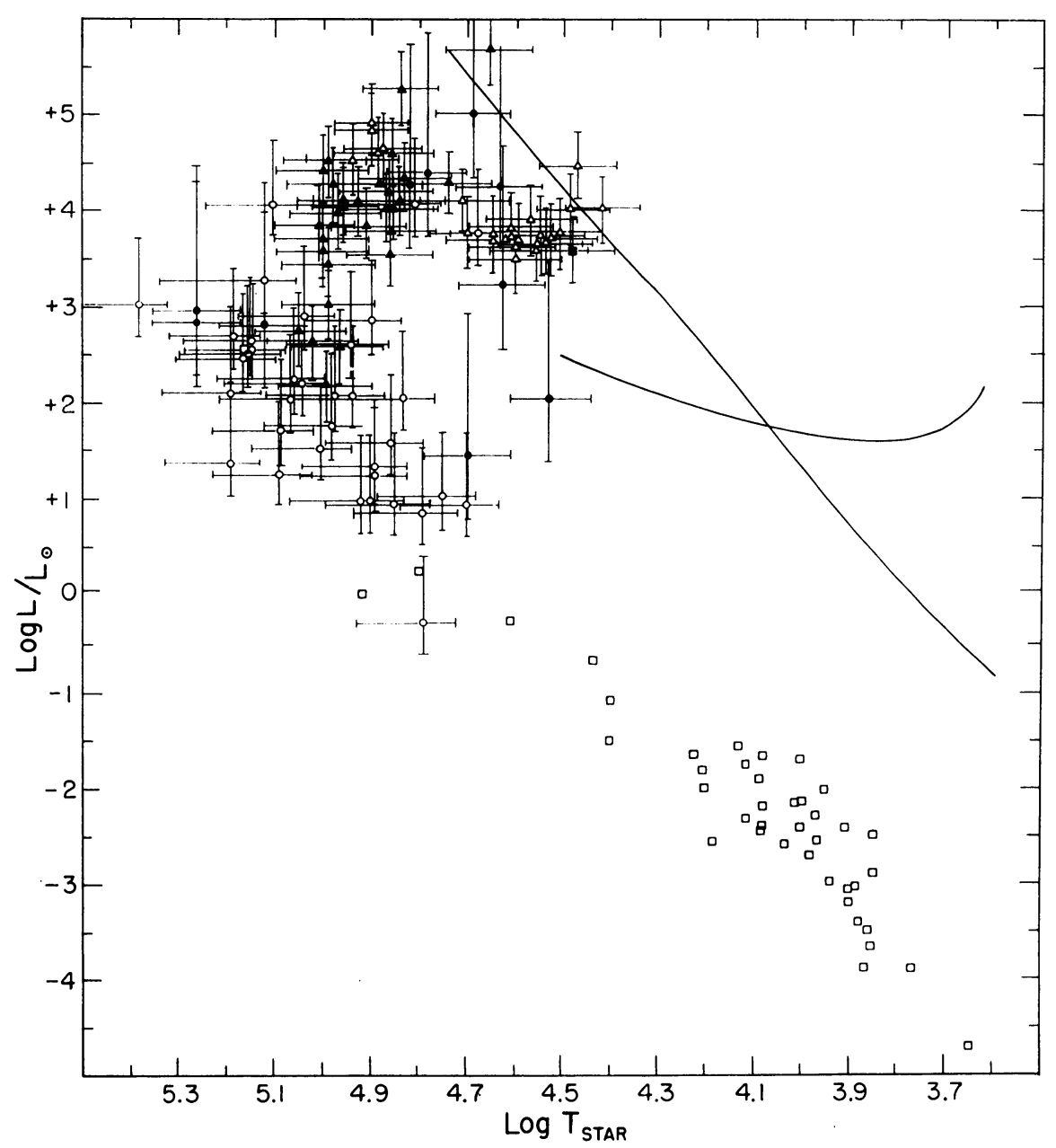

Fig. 1. The composite luminosity-temperature diagram for the nuclei of planetary nebulae and white dwarfs. Open triangles are nuclei in the Magellanic Clouds; filled triangles are optically thin objects with HeII Zanstra temperatures; filled circles are optically thick nebulae; open circles are marginally optically thick; and the filled square is $K 648$ in M 15. Luminosities for the optically thin objects are an average of Seaton's and O' Dell's values. The probable errors are derived from reasonable assumptions of errors of parameters entering the calculations as described in the text.

probably good to a factor of 2-3 and hence represents a rigorous, although sometimes difficult, feature to match in the calculation of theoretical central stars.

It is very interesting that the higher luminosity stars fall along the $\operatorname{limit}(\log L \simeq$ $+4 \cdot 2$ ) where radiation and gas pressure are the same. One would expect the outer parts of the star to be more liable to ejection near this condition, a fact which is even more interesting when one notes that many of the stars of highest luminosity show 
W-R and Of spectral features. These features have disappeared by the time (or perhaps because) the stars have dropped to lower luminosities.

The population along this evolutionary sequence, in terms of total number of known planetary nebula systems is a continuously decreasing function as the star and nebula becomes fainter. This is because of the rapid $\left(S \sim R_{\text {gas }}^{-5}\right)$ decrease of nebular surface brightness. This lower population is contrary to what one would expect from theoretical models for such stars, where the rate of change of stellar characteristics decreases with time and hence the population in our diagram should increase. The rapidly increasing difficulty of identification as a planetary nebula on present surveys probably more than covers other effects and makes it especially dangerous to infer conclusions about physical processes occurring in this lower region from the low population. Very narrow band-pass emission-line surveys are very important here in the determination of the rate at which the stars pass through this stage immediately before becoming simple white dwarfs. If indeed a significant fraction of all stars now becoming white dwarfs do so by means of the planetary nebula phenomenon, then the existence of faint nebular shells would greatly facilitate their identification, since this is a period of much faster internal cooling than is true in the most common white-dwarf objects.

The study of the planetary nebulae by themselves as a natural phenomenon is a justified and fascinating scientific activity; however, their study assumes a much greater significance when tied together with the study of the Galaxy with its stellar population and with the study of the evolution of stars. Fortunately, it is possible to say quite a bit about the nature of the ultimate source of these systems, but the question of the more immediate precursors is very open.

There are several qualitative considerations that lead one to inspect the rate of production of the planetary nebulae and their possible origin. The total number of identified planetaries now exceeds one thousand (Perek and Kohoutek, 1967), a number that, although small, maintains a particular significance when one considers that the lifetime of the phenomenon is only a few tens of thousands of years, indicating that the production rate of the planetary nebulae in the total Galaxy is quite high. The second remarkable general feature is the rather clear evidence that once a star has entered the state giving rise to the nebular phenomenon, that it is a one-way path towards the white-dwarf state - indicating that this arises at the end of the evolutionary sequence for at least some stars.

It is possible to discuss the rate of formation of possible precursors to the planetary nebula stage without knowing or assuming what the intermediate stages of stellar evolution are, a very great advantage for a general evaluation. The time-scale of the main-sequence stage of stellar evolution is now well known. This feature has been used by Schmidt (1963) to investigate the rate at which stars were formed in the past in our vicinity of the Galaxy in order to explain the present luminosity function and total stellar density. One can then take the best-fitting model from his work and calculate the rate at which stars of various masses are now leaving the main sequence. 
Since these post-main-sequence states are short compared to the main-sequence state, this will also be the rate at which stars are being fed into the final stages of stellar evolution. It is advantageous to work not with a unit volume close to the Sun, but rather with a column of unit area perpendicular to and passing through the Galactic plane in the vicinity of the Sun since this frees one to a limited degree from some uncertainties of the $Z$-distribution of the objects considered, although for purposes of convenience we shall give both. We show in Figure 2 the results from calculations of

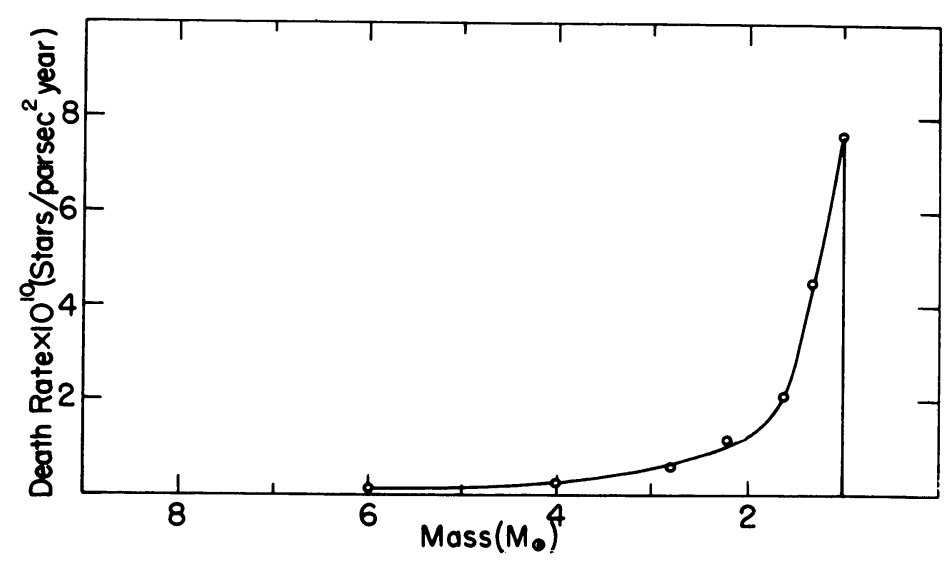

FIG. 2. The death rate of all stars in a unit column perpendicular to the Galactic plane calculated from the Galaxy model of Schmidt (1963). It is seen that the greatest rate of production of stars possibly becoming planetary nebulae occurs at slightly greater than one solar mass. The hydrogen-burning lifetime adopted here is that where it is equal to the age of the Galaxy for a $1.0 M_{\odot}$ star.

the death rate of main-sequence stars from the best-fitting models of Schmidt - where the rate per unit mass is shown. The planetary nebula birth rates for Cahn's (1968) and O'Dell's distance scales are shown in Table 2.

It is quite obvious that upon O'Dell's distance scale, one would require most of the stars evolving from the main sequence - primarily those of close to solar mass due to the much greater rate there - to pass through the planetary-nebula stage. The space velocities of the planetary nebulae argue that most of them belong to a kinematically

\section{Table 2}

Density and Rate of Formation of Planetary Nebulae

$\begin{array}{rlr}\text { Local Density }= & 1.4 \times 10^{-8} \text { planetary nebulae } / \mathrm{parsec}^{3} \\ \text { Local Columnar Frequency }= & 8.1 \times 10^{-6} \text { planetary nebulae } / \mathrm{parsec}^{2} \\ \text { Lifetime } & 35000 \text { years } \\ \text { Rate of Production }= & 3.9 \times 10^{-13} \text { planetary nebulae } / \mathrm{parsec}^{3} \text { year }\left(\text { O'Dell) }^{\prime}\right) \\ & 4.6 \times 10^{-13} \text { planetary nebulae } / \mathrm{parsec}^{3} \text { year (Cahn) } \\ & 2.3 \times 10^{-10} \text { planetary nebulae } / \mathrm{parsec}^{2} \text { year (O'Dell) }\end{array}$


old component of the Galaxy, although not the very oldest, so that there are no inconsistencies with this argument of most low-mass stars passing through this state. The Harman and Seaton scale requires only a small fraction of all evolving stars to pass through this state, although even there the observed kinematics argue for an origin from a star originally not much greater than a solar mass. Neither of these rates of formation are sufficient to account for the observed total density of white dwarfs in the solar neighborhood if continued for $5 \times 10^{9}$ years. However, there is neither compelling reason to think that the rate of production has been constant over such a time interval nor to think that all white dwarfs have come through this phenomenon. It is more critical a test to compare rates of formation of the two classes of objects (cf. Weidemann's paper, below, p. 423).

Speculation about the nature of the immediate progenitors of the planetary nebulae has covered many years and many authors. So many, in fact, that I shall restrict my comments here to a brief condensation of the ideas of several workers in the last few years. Stellar evolution of solar mass stars is reasonably well understood through the red-giant phase and possibly the horizontal branch. It is certainly dangerous to assume $a$ prior $i$ that planetaries are formed soon after this as there may exist several intermediate stages - so that linkage directly to these early evolutionary states is dangerous. In addition, there is so much left out of stellar interior theory (rotation, magnetic fields, etc.) that may become increasingly important in the later stages that additional caution about tying objects together is necessary. The safest procedure is probably to go forward from known stellar states and back in time from the planetary nebula state.

It is very interesting that we pick up the planetary nebulae when they have sizes of about 0.04 parsec radius. This poses a very interesting question of identification. Do their characteristics differ so much when smaller that they do not meet the usual criteria for classification as planetaries and are assigned to some other phenomenon or class of objects - or is the evolution of the nebulae so rapid that relatively few would be expected - or are the nebula essentially formed with this size (that is, perhaps the gas is not ionized before a time corresponding to about this size)? It is hard to believe that the very early evolution in the $2000 \mathrm{AU}$ to 0.04 parsec range could proceed much faster than the later stages, because of the small importance of the gravitational field of the system. Abell and Goldreich (1966) have pointed out that the very earliest stages probably do go very rapidly, but this is earlier than we consider here. The possibility of the star heating up so that photo-ionization occurs at a radius of 0.04 parsec certainly exists, but is difficult to test and will not be further discussed here.

Projection backwards of the observed nebulae would indicate that the immediately preceding stage should be characterized by a very large optical depth in the Lyman continuum and the existence of a mostly neutral outer shell. The high densities expected would suppress the emission of certain forbidden lines (such as 23727 ) by collisional de-excitation and enhance emission from auroral with respect to nebular 
transitions, e.g., $\lambda 4363 / \lambda 5007$. [Fe II] lines would also come up at the higher densities. The Balmer series would also be affected since the greater density would more than compensate for the shorter path length, so that an appreciable optical depth in the lower members of the Balmer series would exist. The nature of the expected continuum radiation is rather uncertain. If the hot star remains dominant, then it would be expected to be quite blue, corresponding to about a B spectral type. However, if a model such as that proposed by Sobolev is valid, then the nebular continuous emission should dominate, with its corresponding red color.

There are a few unclassified objects that generally satisfy these expected conditions for the proto-planetary. The most important two are Minkowski 1-2 and $\mathrm{MH} \alpha$ 328-116. M 1-2 (VV8) was originally identified as a stellar planetary nebula and from its spectrum was inferred to have a very high nebular density by several investigators (Razmadze, 1960). Spectra of the continuum (O'Dell, 1966) revealed the surprising fact that the absorption-line spectrum corresponds approximately to that of a G-type supergiant star, while its continuum flux distribution does not fit that expected for a combination late and early-type binary even though there is a definite ultraviolet continuum present. $\mathrm{MH} \alpha$ 328-116 is an object, originally found as a sixteenth-magnitude Me star, that has undergone an abrupt rise to eleventh magnitude (FitzGerald et al., 1966; O'Dell, 1967). The spectrum indicates Balmer self-absorption (as does M 1-2), very high densities and an observed underlying continuum that is quite flat in wavelength interval - which becomes quite blue after correction for interstellar reddening effects. There do not seem to be any satisfactory spectra of the continua following the outburst although this is a very important feature. It is tempting to associate both of these objects with the proto-planetaries but there are many problems - one of the most important being the question of the origin of the G supergiant spectrum in M 1-2 and the previously found $\mathrm{M}$-spectrum in $\mathrm{MH} \alpha 328-116$. More as a question than a solution, we should like to ask if these late spectra are formed in the neutral outer shell, following the mechanism roughly outlined by Sobolev (1960).

If one looks for a class of objects generally satisfying the prescribed conditions for a proto-planetary, one might look to the symbiotic stars. Although a very definite fraction of this class of stars is binary in nature, an appreciable fraction is probably very similar to the two objects described above and may be single stars with extended outer envelopes surrounded by neutral material. The emission-line shifts are comparable to those found in the planetary nebulae and the nature of the continuum is very uncertain, although an ultraviolet source is definitely within the system. There is an urgent need for continuous spectrographic and spectrophotometric observations of this very important class of objects along the lines of the pioneering efforts of Boyarchuk, Gershberg, and others (Belyakina et al., 1963).

In summary, we can say that by careful selection of data and techniques, it is possible to bring together a rather comprehensive picture of the rapid evolution of the central stars of the planetary nebulae. Like many other areas, we have raised many 
additional questions to be answered, paramount among them being that of the linkage to other known states of stellar evolution.

\section{References}

Abell, G.O. (1966) Astrophys. J., 144, 259.

Abell, G. O., Goldreich, P. (1966) Publ. astr. Soc. Pacific, 78, 232.

Aller, L.H. (1956) Gaseous Nebulae, Chapman and Hall, London, pp. 201-231.

Bautz, L.P. (1968) Astr. J., 73, 54.

Belyakina, T.S., Boyarchuk, A. A., Gershberg, R.E. (1963) Publ. Crimean astrophys. Obs., 30, 25.

Böhm, K.H., Deinzer, W. (1965) Z. Astrophys., 61, 1.

Cahn, J.H. (1968) in the present volume, p. 44.

Capriotti, E.R., Daub, C.T. (1960) Astrophys. J., 132, 677.

Collins, G. W. II, Daub, C. T., O’Dell, C. R. (1961) Astrophys. J., 133, 471.

Deinzer, W. (1967) Z. Astrophys. (in press).

FitzGerald, M.P., Houk, N., McCluskey, S.W., Hoffleit, D. (1966) Astrophys. J., 144, 1135.

Gebbie, K. B., Seaton, M.J. (1963) Nature, 199, 580.

Greenstein, J.L., Minkowski, R.L. (1964) Astrophys. J., 140, 1601.

Harman, R.J., Seaton, M.J. (1966) Mon. Not. R. astr. Soc., 132, 15.

L'Ecuyer, Jean (1966) Astrophys. J., 146, 845.

Liller, W. (1955) Astrophys. J., 122, 240.

O’Dell, C.R. (1962) Astrophys. J., 135, 371.

O'Dell, C.R. (1963a) Astrophys. J., 138, 67.

O'Dell, C.R. (1963b) Astrophys. J., 138, 293.

O'Dell, C.R.(1966) Astrophys. J., 145, 487.

O'Dell, C.R. (1967) Astrophys. J., 149, 373.

O'Dell, C. R., Peimbert, M., Kinman, T.D. (1964) Astrophys. J., 140, 119.

Perek, L., Kohoutek, L. (1967) Atlas of Planetary Nebulae.

Razmadze, N. A. (1960) Astr. J. Soviet Union, 37, 342.

Rose, W. K. (1966) Astrophys. J., 144, 1001.

Schmidt, M. (1963) Astrophys. J., 137, 758.

Seaton, M.J., Osterbrock, D.E. (1957) Astrophys. J., 125, 66.

Seaton, M.J. (1966) Mon. Not. R. astr. Soc., 132, 347.

Shaviv, G. (1967) Astr. J., 72, 319.

Shklovsky, I.S. (1956) Astr. J. Soviet Union, 33, 315.

Sobolev, V.V. (1960) Moving Envelopes of Stars, S. Gaposchkin, translator, Harvard University

Press, Cambridge, Mass., pp. 82-98.

Vila, S.C. (1966) Astrophys. J., 146, 437.

Würm, K., Singer, O. (1952) Z. Astrophys., 30, 387.

Webster, L. B. (1967) private communication.

Weidemann, V. (1968) in the present volume, p. 423.

Zanstra, H. (1926) Phys. Rev., 27, 644.

\section{DISCUSSION}

Böhm: Is it correct to say that stars having Wolf-Rayet spectral type lie always closest to the instability limit due to the action of radiation pressure in the atmosphere? I had the impression that W-R stars occur over a wide range of the evolutionary sequence up to $T_{\text {eff }} \simeq 1.0 \times 10^{5}{ }^{\circ} \mathrm{K}$, where the Harman-Seaton sequence is already relatively far from the instability line.

$O$ 'Dell: When one considers the uncertainties in the temperatures and luminosities as given here, the statement that W-R spectral types always lie close to the instability limit is more descriptive.

Seaton: Highly advanced, optically thick, planetaries may have approximately constant radii of the ionized region. Did you consider the possible use of this result in getting distances? 
O'Dell: No.

Aller: The idea that combination or symbiotic variables are precursors of planetary nebulae has occurred to a number of people. At least it was the basis for the spectrophotometric program on these objects I initiated at Michigan in 1948. The Zanstra-Menzel temperatures of the hot sources in these stars fall at about the right level. The spectral changes appear very disorderly and complicated (as described e.g. by Merrill for BF Cygni). The object must settle down into a nice orderly planetary nebula. The connecting link between the symbiotic stars and the earliest recognized stage of a planetary nebula is yet to be established.

Van Horn: I'd like to ask O'Dell if there are any systematic concentrations of the continuousspectrum stars along the evolutionary track such as he finds for the Wolf-Rayet type nuclei?

$O$ 'Dell: They are concentrated to the lower-luminosity region although not all are of the continuum type there.

Savedoff: What is the reason for difference between your quoted $L=10^{4 \cdot 2}$ and my estimate of $L=10^{4} M /(1+X)$ based on electron scattering?

Deinzer: The difference is probably due to chemical composition and mass. I used $0.4 M_{\odot}$ and a chemical composition of population II. The limiting luminosity is then at about $10^{4 \cdot 2}$.

Westerlund: Miss Webster has observed a large number of galactic planetary nebulae in the Southern hemisphere. Several of those are in Norma with low reddening. She used narrow-band interference filters; in the continuum the wavelengths were 5300,4200 , and 3500 . The nuclei are found near the hot end of the main-sequence relation in the two-color diagram and along the blackbody line. 